\title{
The association between copper transporters and the prognosis of cancer patients undergoing chemotherapy: a meta-analysis of literatures and datasets
}

\author{
Si Sun ${ }^{1, *}$, Jing $\mathrm{Cai}^{1, *}$, Qiang Yang ${ }^{1}$, Simei Zhao ${ }^{1}$ and Zehua Wang ${ }^{1}$ \\ ${ }^{1}$ Department of Obstetrics and Gynecology, Union Hospital, Tongji Medical College, Huazhong University of Science and \\ Technology, Wuhan, Hubei, People's Republic of China \\ * These authors have contributed equally to this study \\ Correspondence to: Zehua Wang, email: zehuawang@163.net
}

Keywords: CTR 1, ovarian cancer, lung cancer, platinum-based chemotherapy, meta-analysis

Received: June 30, 2016

Accepted: December 01, 2016

Published: December 12, 2016

\section{ABSTRACT}

Copper transporter 1 (CTR1), copper transporter 2 (CTR2), copper-transporting p-type adenosine triphosphatase 1 and 2 (ATP7A and ATP7B) are key mediators of cellular cisplatin, carboplatin and oxaliplatin accumulation. In this meta-analysis, we aimed to evaluate the relation of CTR1, CTR2, ATP7A and ATP7B to overall survival (OS), progression-free survival (PFS), disease-free survival (DFS) and treatment response (TR) of cancer patients who received chemotherapy based on published literatures, the Gene Expression Omnibus (GEO) and the Cancer Genome Atlas (TCGA) datasets. Hazard ratios (HRs) and odds ratios (ORs) were pooled using random-effect models. Subgroup analysis and sensitivity analysis were conducted; heterogeneity and publication bias were assessed. Twelve literatures and eight datasets with 2149 patients were included. Our results suggested that high CTR1 expression was associated with favorable OS, PFS, DFS and TR in cancer patients who underwent chemotherapy with acceptable heterogeneity. The relationship of CTR1 to cancer prognosis remained significant in the subgroup of patients who underwent platinumbased chemotherapy, the patients with ovarian cancer and those with lung cancer. The significance of these relationships was not influenced by geological region of publication, data origin or detection method. However, there was no evidence for relation of CTR2, ATP7A or ATP7B to OS, PFS, DFS or TR. Test of publication bias and sensitivity analysis suggested a robustness of all the summary effect estimates. In conclusion, high CTR1 level predicts prolonged survival and enhanced response to chemotherapy in cancer patients who underwent chemotherapy and CTR1 might be a potential target to circumvent chemotherapy resistance.

\section{INTRODUCTION}

Platinum-based chemotherapy remains a first-line treatment of various epithelial malignancies including lung, bladder, head and neck and gynecological cancers for over 30 years. Although over half of the patients respond well to platinum-based chemotherapy, relapse and drugresistance are major limitations for successful treatment of cancers. The platinum drugs exert cytotoxicity mainly through binding DNA to form crosslinks, which interfere normal DNA activities and induce cellular apoptosis [1].
However in resistant cancer cells, decreased cellular drug accumulation impairs the formation of DNA-platinum abducts. Low platinum concentration in tumor tissue is significantly associated with reduced tumor response [2, $3]$. Therefore, drug accumulation is identified as a key process that governs response to chemotherapy in cancer.

Precedent evidence suggested that platinum drugs entered cancer cells through passive diffusion because the time-linear influx of platinum did not reach saturation at relevant drug concentration, was not inhibited by any structural analogous nor was it dependent on an optimal $\mathrm{pH}$ [4]. According to recent studies however, 
Table 1: The characteristics of the studies included in the meta-analysis.

\begin{tabular}{|c|c|c|c|c|c|c|c|}
\hline \begin{tabular}{|l|}
$\begin{array}{l}\text { Author } \\
\text { (Year) }\end{array}$ \\
\end{tabular} & Country & \begin{tabular}{|l|}
$\begin{array}{l}\text { Type of } \\
\text { transporter }\end{array}$ \\
\end{tabular} & \begin{tabular}{|l|} 
Case/ \\
Control \\
\end{tabular} & \begin{tabular}{|l|} 
Data \\
origin
\end{tabular} & Cancer type & \begin{tabular}{|l|} 
End- \\
points
\end{tabular} & \begin{tabular}{|l|}
$\begin{array}{l}\text { Detection } \\
\text { methods }\end{array}$ \\
\end{tabular} \\
\hline $\begin{array}{l}\text { Hsu } \\
(2015)\end{array}$ & China & CTR1 & $40 / 40$ & Study & Ovarian cancer & PFS & IHC \\
\hline $\begin{array}{l}\text { Yang } \\
(2015)\end{array}$ & China & $\begin{array}{l}\text { CTR1 } \\
\text { ATP7A } \\
\text { ATP7B } \\
\end{array}$ & \begin{tabular}{|l|}
$32 / 22$ \\
$20 / 34$ \\
$15 / 39$ \\
\end{tabular} & Study & Lung cancer & OS, TR & $\mathrm{IHC}$ \\
\hline $\begin{array}{l}\text { TCGA } \\
(2015)\end{array}$ & USA & $\begin{array}{l}\text { CTR1 } \\
\text { CTR2 ATP7A } \\
\text { ATP7B }\end{array}$ & \begin{tabular}{|l|}
$174 / 331$ \\
$129 / 376$ \\
$324 / 181$ \\
$174 / 331$ \\
\end{tabular} & Dataset & Ovarian cancer & $\begin{array}{l}\text { OS, PFS, } \\
\text { TR }\end{array}$ & Gene array \\
\hline $\begin{array}{l}\text { Ogane } \\
(2013)\end{array}$ & Japan & CTR1 & $30 / 17$ & Study & Endometrial cancer & OS, DFS & $\mathrm{IHC}$ \\
\hline $\begin{array}{l}\begin{array}{l}\text { Katagiri } \\
(2013)\end{array} \\
\end{array}$ & Japan & ATP7B & $25 / 61$ & Study & Ovarian cancer & OS, PFS & $\mathrm{IHC}$ \\
\hline $\begin{array}{l}\text { Chen } \\
(2012)\end{array}$ & China & $\begin{array}{l}\text { CTR1 ATP7A } \\
\text { ATP7B }\end{array}$ & $\begin{array}{l}37 / 17 \\
26 / 28 \\
40 / 14 \\
\end{array}$ & Study & Lung cancer & $\begin{array}{l}\text { OS, PFS, } \\
\text { TR }\end{array}$ & IHC \\
\hline $\begin{array}{l}\mathrm{Li} \\
(2012) \\
\end{array}$ & China & ATP7A & $37 / 52$ & Study & Lung cancer & OS & $\mathrm{IHC}$ \\
\hline $\begin{array}{l}\text { LeeJK } \\
(2012)\end{array}$ & USA & $\begin{array}{l}\text { CTR1 } \\
\text { CTR2 ATP7A } \\
\text { ATP7B }\end{array}$ & $\begin{array}{l}15 / 43 \\
45 / 13 \\
45 / 13 \\
18 / 40 \\
\end{array}$ & Dataset & Ovarian cancer & $\begin{array}{l}\text { OS, PFS, } \\
\text { TR }\end{array}$ & Gene array \\
\hline $\begin{array}{l}\text { LeeYY } \\
(2011) \\
\end{array}$ & Korea & \begin{tabular}{|l|} 
CTR1 \\
CTR2 \\
\end{tabular} & $\begin{array}{l}20 / 20 \\
20 / 20 \\
\end{array}$ & Study & Ovarian cancer & PFS, TR & qrt-PCR \\
\hline $\begin{array}{l}\text { Birrer } \\
(2011)\end{array}$ & USA & $\begin{array}{l}\text { CTR1 } \\
\text { CTR2 ATP7A } \\
\text { ATP7B }\end{array}$ & $\begin{array}{l}89 / 95 \\
57 / 127 \\
70 / 114 \\
122 / 62 \\
\end{array}$ & Dataset & Ovarian cancer & OS, PFS & Gene array \\
\hline $\begin{array}{l}\text { Mateescu } \\
(2011)\end{array}$ & France & $\begin{array}{l}\text { CTR1 } \\
\text { CTR2 ATP7A } \\
\text { ATP7B }\end{array}$ & $\begin{array}{l}48 / 45 \\
68 / 25 \\
49 / 44 \\
26 / 67 \\
\end{array}$ & Dataset & Ovarian cancer & $\begin{array}{l}\text { OS, PFS, } \\
\text { TR }\end{array}$ & Gene array \\
\hline $\begin{array}{l}\text { Tsao } \\
(2010)\end{array}$ & Canada & $\begin{array}{l}\text { CTR1 } \\
\text { CTR2 ATP7A } \\
\text { ATP7B }\end{array}$ & \begin{tabular}{|l|}
$30 / 20$ \\
$24 / 26$ \\
$29 / 21$ \\
$27 / 23$ \\
\end{tabular} & Dataset & Lung cancer & OS & Gene array \\
\hline $\begin{array}{l}\text { Martinez- } \\
\text { Balibrea } \\
(2009) \\
\end{array}$ & Spain & ATP7B & $16 / 34$ & Study & Colorectal cancer & PFS, TR & qrt-PCR, IHC \\
\hline $\begin{array}{l}\text { Denkert } \\
(2009)\end{array}$ & Germany & $\begin{array}{l}\text { CTR1 } \\
\text { CTR2 ATP7A } \\
\text { ATP7B }\end{array}$ & \begin{tabular}{|l|}
$24 / 54$ \\
$43 / 35$ \\
$35 / 43$ \\
$30 / 48$ \\
\end{tabular} & Dataset & Ovarian cancer & OS, PFS & Gene array \\
\hline $\begin{array}{l}\text { Tothill } \\
(2008)\end{array}$ & Australia & \begin{tabular}{ll|} 
CTR1 \\
CTR2 ATP7A \\
ATP7B
\end{tabular} & $\begin{array}{l}118 / 124 \\
183 / 59 \\
95 / 147 \\
117 / 125 \\
\end{array}$ & Dataset & Ovarian cancer & OS, PFS & Gene array \\
\hline $\begin{array}{l}\text { Aida } \\
(2005)\end{array}$ & Japan & ATP7B & $19 / 32$ & Study & Endometrial cancer & OS, DFS & $\mathrm{IHC}$ \\
\hline $\begin{array}{l}\text { Nevins } \\
(2005)\end{array}$ & USA & $\begin{array}{l}\text { CTR1 } \\
\text { CTR2 ATP7A } \\
\text { ATP7B }\end{array}$ & $\begin{array}{l}30 / 85 \\
76 / 39 \\
71 / 44 \\
85 / 30\end{array}$ & Dataset & Ovarian cancer & OS & Gene array \\
\hline $\begin{array}{l}\text { Nakayama } \\
(2004)\end{array}$ & Japan & ATP7B & $37 / 67$ & Study & Ovarian cancer & OS, TR & $\mathrm{IHC}$ \\
\hline $\begin{array}{l}\begin{array}{l}\text { Samimi } \\
(2003)\end{array} \\
\end{array}$ & USA & ATP7A & $25 / 29$ & Study & Mixed cancers & OS & IHC \\
\hline $\begin{array}{l}\text { Nakayama } \\
(2002)\end{array}$ & Japan & ATP7B & $36 / 46$ & Study & Ovarian cancer & OS & qrt-PCR \\
\hline
\end{tabular}

IHC: Immunochemistry; qRT-PCR: Real-time polymerase chain reaction; OS: Overall survival; PFS: Progression-free survival; DFS: Disease-free survival; TR: Treatment response 
platinum accumulation was predominantly determined by transporters, which mediate either active or passive transportation of the drug [5]. Early evidence revealed that cellular cisplatin concentration was associated with cellular copper concentration $[6,7]$. Studies from several independent laboratories revealed that copper metabolic pathways were involved in influx and efflux of platinum. CTR1 (copper transporter 1), CTR2 (copper transporter 2), ATP7A (copper-transporting p-type adenosine triphosphatase 1) and ATP7B (copper-transporting p-type adenosine triphosphatase 2) are four major copper transporters which mediate homeostasis and copper metabolism. With two conserved copper/cisplatin binding motifs, CTR1 facilitates copper and platinum to traverse through cellular membrane. CTR2 regulates copper/ cisplatin storage by interacting and truncating CTR1 to smaller fragment, which is deprived of the ability to transport copper and cisplatin [8]. ATP7A sequesters intracellular platinum complex distribution and facilitates efflux of platinum complex. The turnover of ATP7B between trans Golgi network and cell membrane through endosomal/lysomal vesicle trafficking regulates cellular copper hemostasis by eliminating excess copper, which is also involved in cellular platinum elimination [9]. In addition to cisplatin, carboplatin and oxaliplatin are also substrates of these copper transporters [10-12] (Figure 1). Preclinical studies demonstrated that CTR1, CTR2, ATP7A and ATP7B expression regulate cellular response to cisplatin $[8,13-15]$. However, the relationship between these copper transporters and the prognosis of cancer patients who received chemotherapy are inconclusive.

Therefore, we conducted a meta-analysis of published literatures as well as datasets to assess the association between the expression of copper transporters and the prognosis in cancer patients who underwent chemotherapy. To obtain more detailed information, subgroup analyses stratified by cancer type, chemotherapy regimen, geological region, data origin and detection method were conducted.

\section{RESULTS}

\section{Study characteristics}

A total of 1793 literatures were identified from electronic search. Full texts of thirty publications were retrieved after reviewing titles and abstracts. After exclusion of 18 literatures (Table S1), finally, twelve studies [16-27] and eight datasets (GSE3149, GSE9891, GSE26712, GSE30161, GSE14764, GSE26193, GSE14814 and TCGA) [28-35] with 2149 cases met the inclusion criteria (Figure 2 and Table 1).

\section{Copper transporters and survival}

Eleven studies evaluated the relationship between CTR1 expression and OS of cancer patients who received chemotherapy, and ten studies evaluated PFS/DFS. High CTR1 expression was correlated with a favorable OS and PFS in cancers (for OS, HR $=0.64,95 \%$ CI: $0.50-0.82$, $P=0.000$; for PFS, HR $=0.65,95 \%$ CI: $0.57-0.75, P=$ 0.000 ) (Figure 3 and Figure 4). The heterogeneities across studies included were moderate and acceptable (for OS, $I^{2}=47.3 \%$; for PFS, $I^{2}=0.0 \%$ ). However, there was no significant relation of the expression of CTR2, ATP7A and ATP7B to OS or PFS/DFS (Figure 5-10).

\section{Subgroup analysis of survival}

Subgroup analysis was performed by stratifying data according to cancer type, chemotherapy regimen, geographic region, data origin and detection method. High CTR1 expression was significantly correlated with a favorable OS in the subgroups of "ovarian cancer" (HR $=0.71,95 \%$ CI: $0.57-0.90, P=0.004)$, lung cancer" (HR $=0.31,95 \%$ CI: $0.17-0.58, P=0.000)$, "platinum-based" (HR $=0.63,95 \%$ CI: $0.49-0.81, P=0.000)$, "America" $(\mathrm{HR}=0.68,95 \%$ CI: 0.82-0.88, $P=0.003)$, "Asia" (HR $=0.34,95 \%$ CI: $0.16-0.73, P=0.006)$, "dataset" $(\mathrm{HR}=$ $0.86,95 \%$ CI: $0.53-0.87, P=0.002)$, "literature" $(\mathrm{HR}=$ $0.34,95 \%$ CI: $0.16-0.73, P=0.006$ ), "gene array" (HR $=0.86,95 \%$ CI: $0.53-0.87, P=0.002)$ and "IHC" $(\mathrm{HR}=$ $0.34,95 \%$ CI: $0.16-0.73, P=0.006)$. Similarly, high CTR1 expression was associated with an improved PFS/DFS in the subgroups of "ovarian cancer" (HR $=0.67,95 \% \mathrm{CI}$ : $0.58-0.76, P=0.000$ ), "platinum-based" ( $\mathrm{HR}=0.63,95 \%$ CI: $0.54-0.73, P=0.000)$, "America" (HR $=0.75,95 \% \mathrm{CI}$ : $0.62-0.90, P=0.002)$, “Asia" (HR $=0.44,95 \%$ CI: 0.31 $0.62, P=0.000)$, "Europe" ( $\mathrm{HR}=0.63,95 \% \mathrm{CI}: 0.44-$ $0.88, P=0.007)$, "dataset" ( $\mathrm{HR}=0.70,95 \% \mathrm{CI}: 0.61-0.81$, $P=0.000)$, "literature" ( $\mathrm{HR}=0.44,95 \%$ CI: $0.31-0.62, P$ $=0.000)$, "gene array" (HR $=0.70,95 \%$ CI: 0.61-0.81, $P=0.000)$, "IHC" (HR $=0.47,95 \%$ CI: $0.26-0.84, P=$ $0.010)$ and "PCR" (HR $=0.42,95 \%$ CI: $0.27-0.65, P=$ $0.000)$ (Table 2). The heterogeneities were moderate $\left(I^{2}\right.$ range, $0.0 \%-49.5 \%$ ) and acceptable. High CTR2 was associated with an unfavorable PFS in the "America" subgroup $(\mathrm{HR}=1.32,95 \% \mathrm{CI}: 1.08-1.62, P=0.007)$, while in the "Europe" subgroup high CTR2 was associated with a favorable OS (HR $=0.61,95 \%$ CI: $0.40-0.93, P=$ 0.020 ) and PFS/DFS (HR $=0.66,95 \%$ CI: $0.45-0.95, P$ $=0.026$ ). No evidence of a significant heterogeneity was found (Table 2). High ATP7B was correlated with a poor PFS in the "literature" subgroup (HR $=2.27,95 \% \mathrm{CI}$ : $1.16-4.42, P=0.016)$ with a significant heterogeneity $\left(I^{2}\right.$ $=69.0)($ Table 3$)$. 


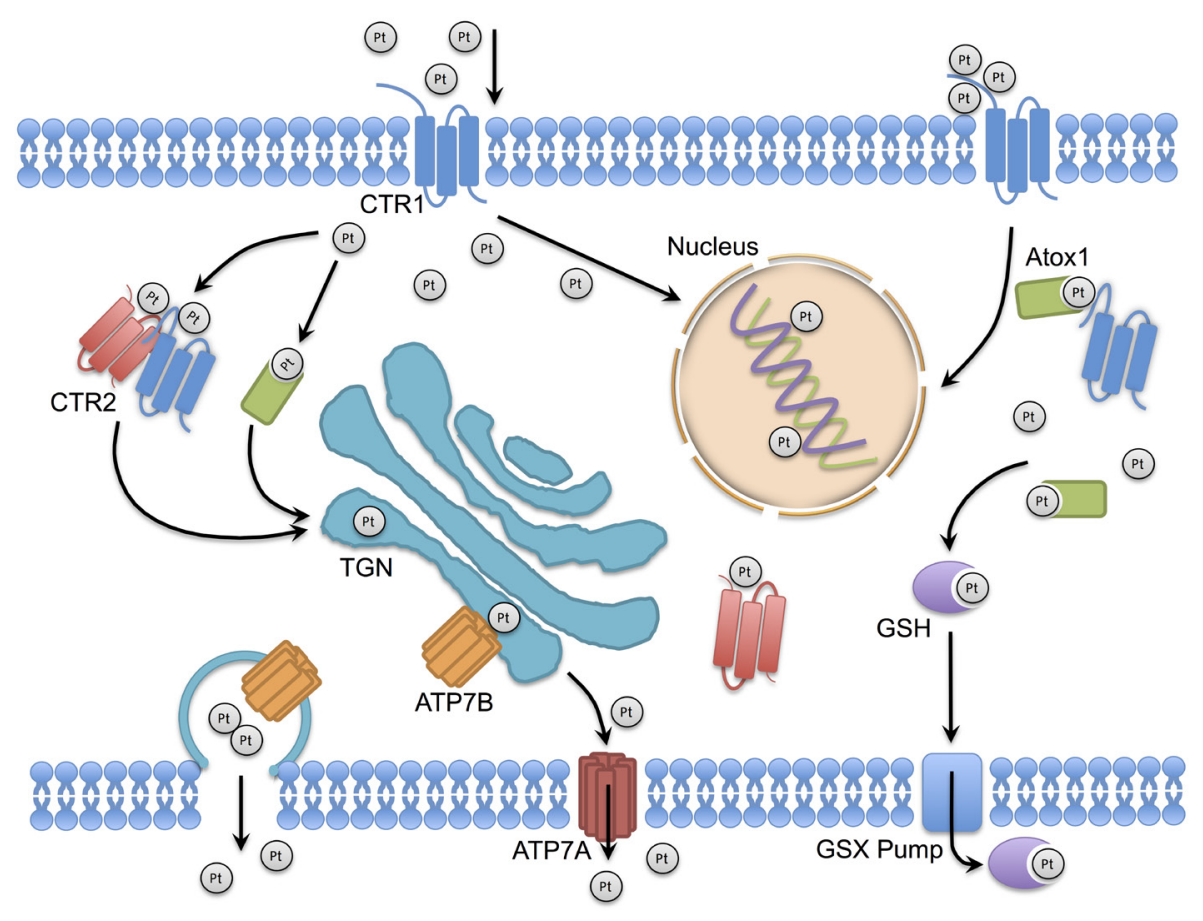

Figure 1: The role of copper homeostasis system in platinum drug transportation and trafficking. Platinum drugs enter the cells by active CTR1 transportation. CTR1 and CTR2 ferry platinum to molecular chaperones, cell organelles and drug targets to proceeding platinum distribution or to initiate biological pathways (such as cytotoxic pathway and detoxication pathway). ATP7A and ATP7B regulate the subcellular distribution of cytosol platinum to trans Golgi network and mediate the removal of cellular platinum complex. CTR1, copper transporter 1; CTR2, copper transporter 2; ATP7A, copper-transporting p-type adenosine triphosphatases 1; ATP7B, copper-transporting p-type adenosine triphosphatases 2; TGN, trans Golgi network; GSH, glutathione; Atox1, human antioxidant protein 1; GS-X pump, glutathione S-conjugate pump.

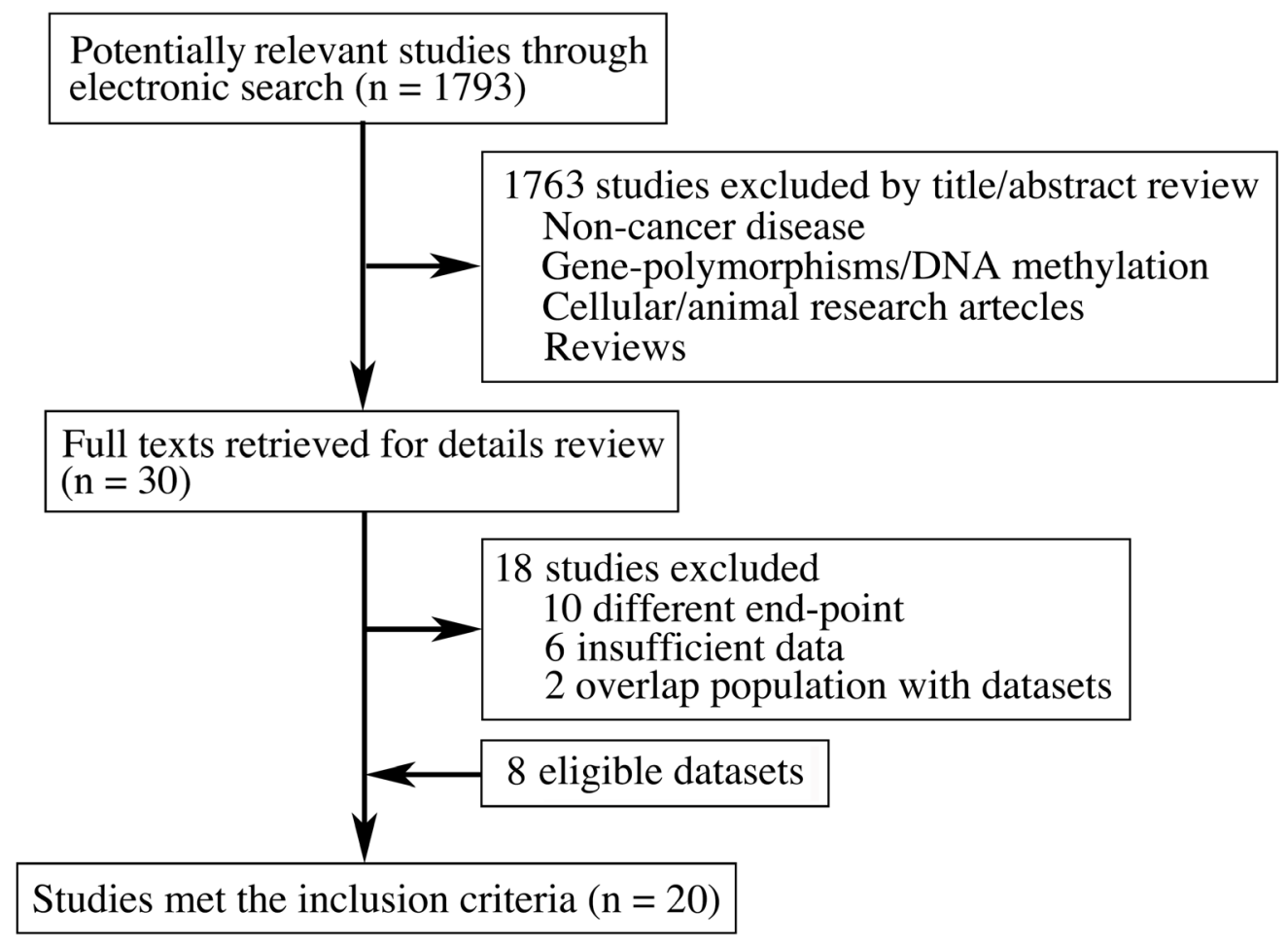

Figure 2: Flowchart of study selection. 


\section{CTR1 and Overall Survival}

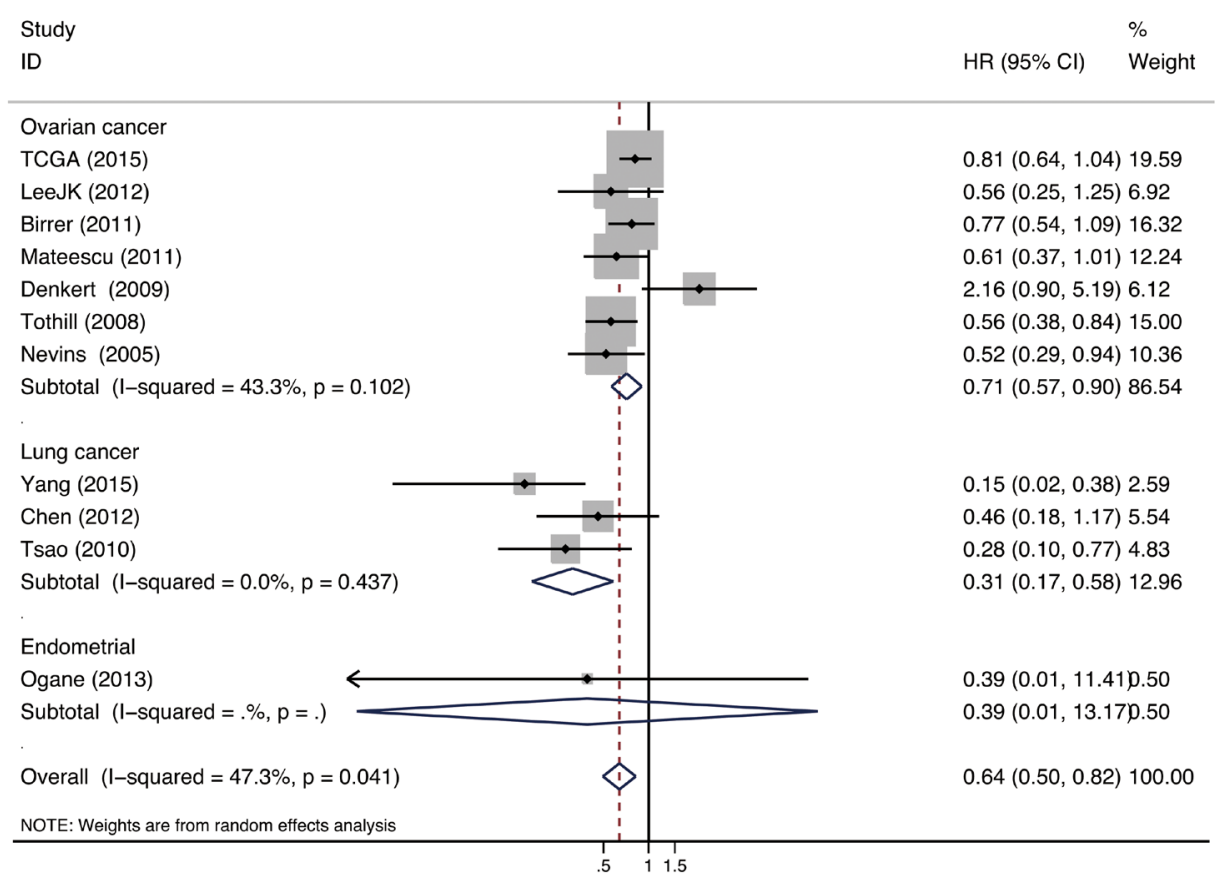

Figure 3: CTR1 and overall survival. Forest plot presents summary hazard ratios (HRs) and $95 \%$ confidence intervals (CIs) of correlation between CTR1 expression and overall survival (OS) in epithelial cancers.

\section{CTR1 and Progression-free Survival/Disease-free Survival}

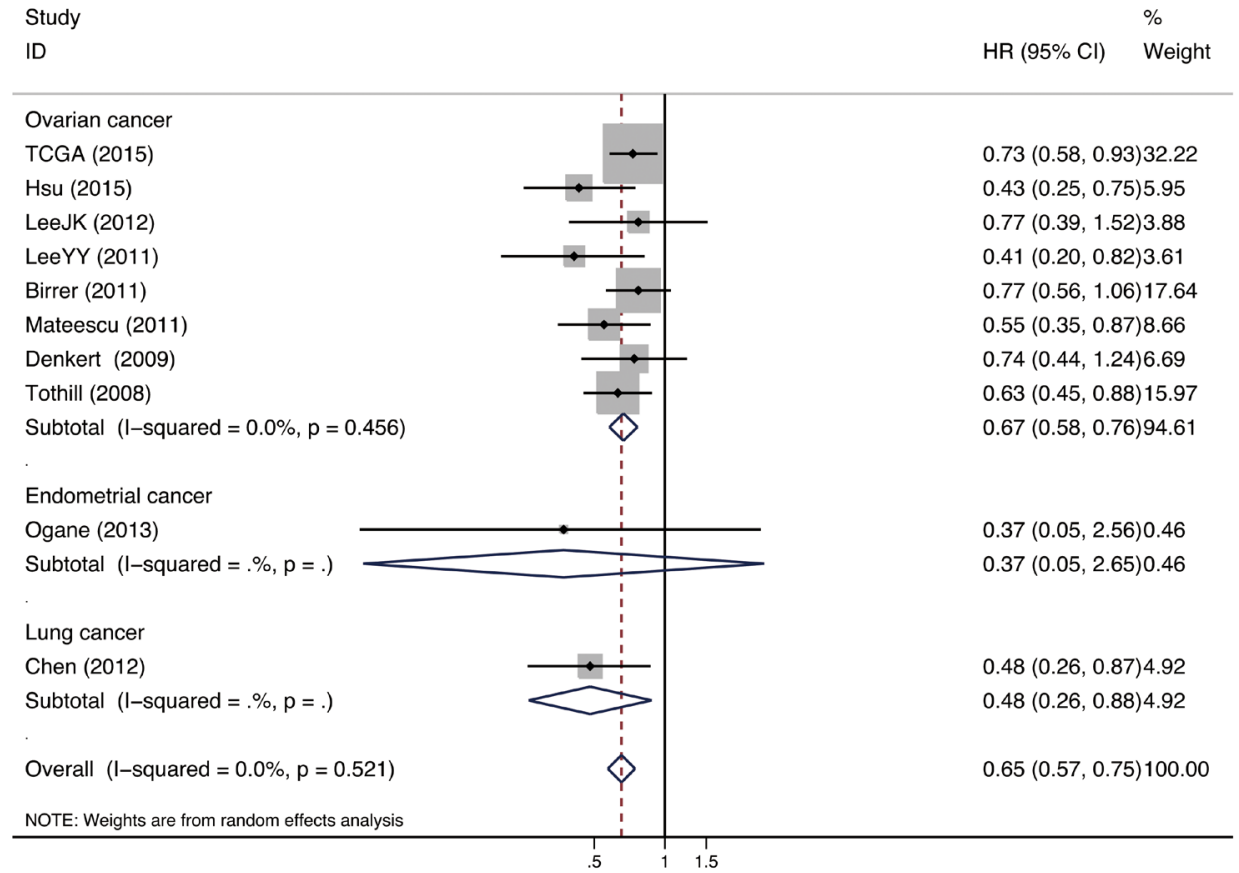

Figure 4: CTR1 and progression-free survival/disease-free survival. Forest plot presents summary hazard ratios (HRs) and 95\% confidence intervals (CIs) of correlation between CTR1 expression and progression free survival/disease-free survival (PFS/DFS in epithelial cancers. 


\section{CTR2 and Overall Survival}

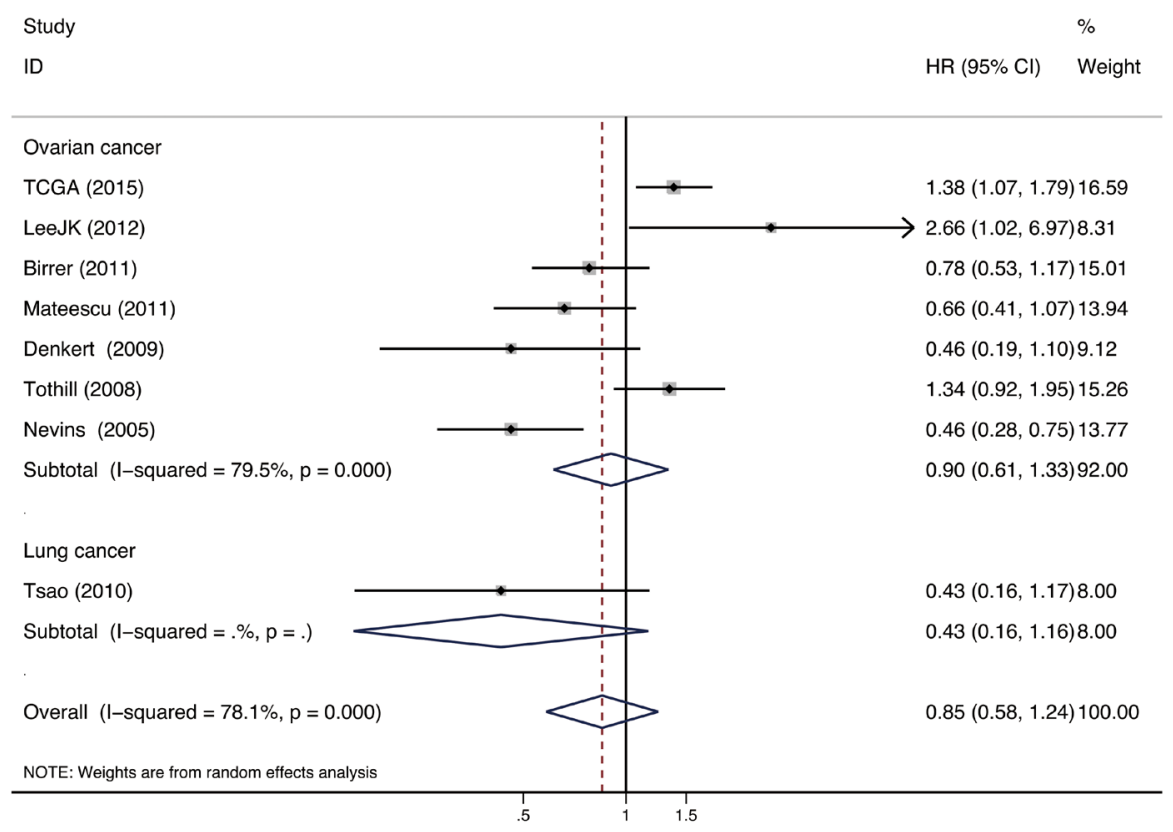

Figure 5: CTR2 and overall survival. Forest plot presents summary hazard ratios (HRs) and 95\% confidence intervals (CIs) of correlation between CTR2 expression and overall survival (OS) in epithelial cancers.

\section{CTR2 and Progression-free Survival/Disease-free Survival}

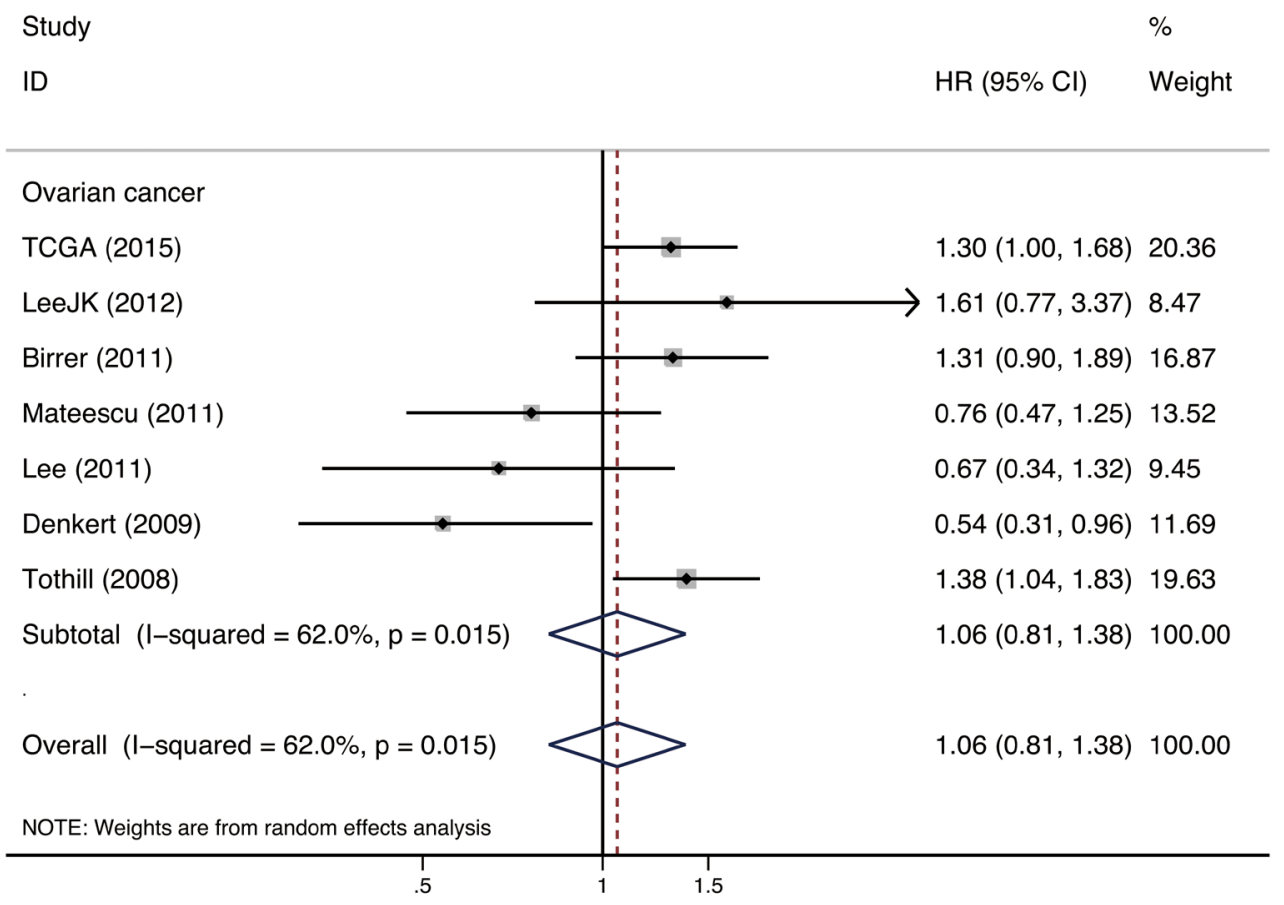

Figure 6: CTR2 progression-free survival/disease-free survival. Forest plot presents summary hazard ratios (HRs) and $95 \%$ confidence intervals (CIs) of correlation between CTR2 expression and progression free survival/disease-free survival (PFS/DFS in epithelial cancers. 
Table 2: Subgroup analyses of CTR1 and CTR2 for survival.

\begin{tabular}{|c|c|c|c|c|c|c|c|c|c|}
\hline & \multirow{2}{*}{ End-point } & \multicolumn{4}{|c|}{ CTR1 } & \multicolumn{4}{|c|}{ CTR2 } \\
\hline & & $\mathbf{N}$ & HR $(95 \% \mathrm{CI})$ & $P$ & $I^{2}(\%)$ & $\mathbf{N}$ & HR $(95 \% \mathrm{CI})$ & $P$ & $I^{2}(\%)$ \\
\hline \multicolumn{10}{|l|}{ Cancer type } \\
\hline \multirow[t]{2}{*}{ Ovarian cancer } & OS & 7 & $0.71(0.57,0.90)$ & 0.004 & 43.3 & 7 & $0.90(0.61,1.33)$ & 0.609 & 79.5 \\
\hline & $\begin{array}{l}\text { PFS/ } \\
\text { DFS }\end{array}$ & 8 & $0.67(0.58,0.76)$ & 0.000 & 0.0 & 7 & $1.06(0.81,1.38)$ & 0.679 & 62.0 \\
\hline \multirow[t]{2}{*}{ Lung cancer } & OS & 3 & $0.31(0.17,0.58)$ & 0.000 & 0.0 & 1 & $0.43(0.16,1.16)$ & 0.096 & - \\
\hline & $\begin{array}{l}\text { PFS/ } \\
\text { DFS }\end{array}$ & 1 & $0.48(0.26,0.88)$ & 0.017 & - & - & - & - & - \\
\hline \multirow[t]{2}{*}{$\begin{array}{l}\text { Endometrial } \\
\text { cancer }\end{array}$} & OS & 1 & $0.39(0.01,13.17)$ & 0.600 & - & - & - & - & - \\
\hline & $\begin{array}{l}\text { PFS/ } \\
\text { DFS }\end{array}$ & 1 & $0.37(0.05,2.65)$ & 0.322 & - & - & - & - & - \\
\hline \multicolumn{10}{|c|}{ Chemotherapy regimen } \\
\hline \multirow[t]{2}{*}{ Platinum-based } & OS & 11 & $0.63(0.49,0.81)$ & 0.000 & 47.1 & 8 & $0.79(0.55,1.12)$ & 0.179 & 72.6 \\
\hline & $\begin{array}{l}\text { PFS/ } \\
\text { DFS }\end{array}$ & 10 & $0.63(0.54,0.73)$ & 0.000 & 11.7 & 7 & $1.11(0.89,1.38)$ & 0.362 & 43.8 \\
\hline \multicolumn{10}{|l|}{ Geographic region } \\
\hline \multirow[t]{2}{*}{ America } & OS & 5 & $0.68(0.52,0.88)$ & 0.003 & 31.9 & 5 & $0.88(0.51,1.54)$ & 0.659 & 83.2 \\
\hline & $\begin{array}{l}\text { PFS/ } \\
\text { DFS }\end{array}$ & 3 & $0.75(0.62,0.90)$ & 0.002 & 0.0 & 3 & $1.32(1.08,1.62)$ & 0.007 & 0.0 \\
\hline \multirow[t]{2}{*}{ Asia } & OS & 3 & $0.34(0.16,0.73)$ & 0.006 & 0.0 & - & - & - & - \\
\hline & $\begin{array}{l}\text { PFS/ } \\
\text { DFS }\end{array}$ & 4 & $0.44(0.31,0.62)$ & 0.000 & 0.0 & 1 & $0.67(0.34,1.32)$ & 0.247 & - \\
\hline \multirow[t]{2}{*}{ Europe } & OS & 2 & $1.09(0.32,3.74)$ & 0.893 & 83.4 & 2 & $0.61(0.40,0.93)$ & 0.020 & 47.9 \\
\hline & $\begin{array}{l}\text { PFS/ } \\
\text { DFS }\end{array}$ & 2 & $0.63(0.44,0.88)$ & 0.007 & 0.0 & 2 & $0.66(0.45,0.95)$ & 0.026 & 0.0 \\
\hline \multirow[t]{2}{*}{ Oceania } & OS & 1 & $0.49(0.33,0.73)$ & 0.004 & - & 1 & $1.34(0.92,1.95)$ & 0.127 & - \\
\hline & $\begin{array}{l}\text { PFS/ } \\
\text { DFS }\end{array}$ & 1 & $0.51(0.38,0.69)$ & 0.007 & - & 1 & $1.38(1.04,1.83)$ & 0.025 & - \\
\hline \multicolumn{10}{|l|}{ Data origin } \\
\hline \multirow[t]{2}{*}{ Dataset } & OS & 8 & $0.86(0.53,0.87)$ & 0.002 & 49.5 & 8 & $0.85(0.58,1.24)$ & 0.403 & 78.1 \\
\hline & $\begin{array}{l}\text { PFS/ } \\
\text { DFS }\end{array}$ & 6 & $0.70(0.61,0.81)$ & 0.000 & 0.0 & 6 & $1.11(0.85,1.45)$ & 0.437 & 61.9 \\
\hline \multirow[t]{2}{*}{ Literature } & OS & 3 & $0.34(0.16,0.73)$ & 0.006 & 0.0 & - & - & - & - \\
\hline & $\begin{array}{l}\text { PFS/ } \\
\text { DFS }\end{array}$ & 4 & $0.44(0.31,0.62)$ & 0.000 & 0.0 & 1 & $0.67(0.34,1.32)$ & 0.247 & - \\
\hline \multirow{3}{*}{$\begin{array}{l}\text { Detection method } \\
\text { Gene array }\end{array}$} & & & & & & & & & \\
\hline & OS & 8 & $0.86(0.53,0.87)$ & 0.002 & 49.5 & 8 & $0.85(0.58,1.24)$ & 0.403 & 78.1 \\
\hline & $\begin{array}{l}\text { PFS/ } \\
\text { DFS }\end{array}$ & 6 & $0.70(0.61,0.81)$ & 0.000 & 0.0 & 6 & $1.11(0.85,1.45)$ & 0.437 & 61.9 \\
\hline \multirow[t]{2}{*}{ IHC } & OS & 3 & $0.34(0.16,0.73)$ & & 0.0 & - & - & - & - \\
\hline & $\begin{array}{l}\text { PFS/ } \\
\text { DFS }\end{array}$ & 2 & $0.47(0.26,0.84)$ & 0.010 & 0.0 & - & - & - & - \\
\hline \multirow[t]{2}{*}{ qRT-PCR } & OS & - & - & - & - & - & - & - & - \\
\hline & $\begin{array}{l}\text { PFS/ } \\
\text { DFS }\end{array}$ & 2 & $0.42(0.27,0.65)$ & 0.000 & 0.0 & 1 & $0.67(0.34,1.32)$ & 0.247 & - \\
\hline
\end{tabular}

P-value for significance of HR. ( $<$ 0.050) HR: hazard ratio; CI: confidence interval ; IHC: immunochemistry; qRT-PCR: real-time polymerase chain reaction

\section{Copper transporters and treatment response}

Six studies evaluated CTR1 expression and TR of cancer patients who received adjuvant chemotherapy. High CTR1 expression was associated with increased TR (OR $=2.99,95 \% \mathrm{CI}: 1.57-5.69, P=0.001)$, especially in the subgroups of "ovarian cancer" $(\mathrm{OR}=2.28,95 \% \mathrm{CI}: 1.15$ $4.54, P=0.019)$, "lung cancer" $(\mathrm{OR}=5.64,95 \% \mathrm{CI}: 2.17$ -
14.65, $P=0.000)$, "Asia" $(\mathrm{OR}=6.39,95 \%$ CI: 2.83-14.41, $P=0.000)$, "dataset" $(\mathrm{OR}=1.61,95 \%$ CI: $1.10-2.37, P=$ $0.015)$, "literature" $(\mathrm{OR}=6.39,95 \% \mathrm{CI}: 2.83-14.41, P=$ $0.000)$, "gene array" $(\mathrm{OR}=1.61,95 \% \mathrm{CI}: 1.10-2.37, P=$ $0.015)$ and "IHC" $(\mathrm{OR}=5.64,95 \% \mathrm{CI}: 2.17-14.65, P=$ $0.000)$ subgroups. The heterogeneities were moderate and acceptable. No evidence for a correlation between CTR2, ATP7A or ATP7B expression and TR was found (Table 4). 


\section{ATP7A and Overall Survival}

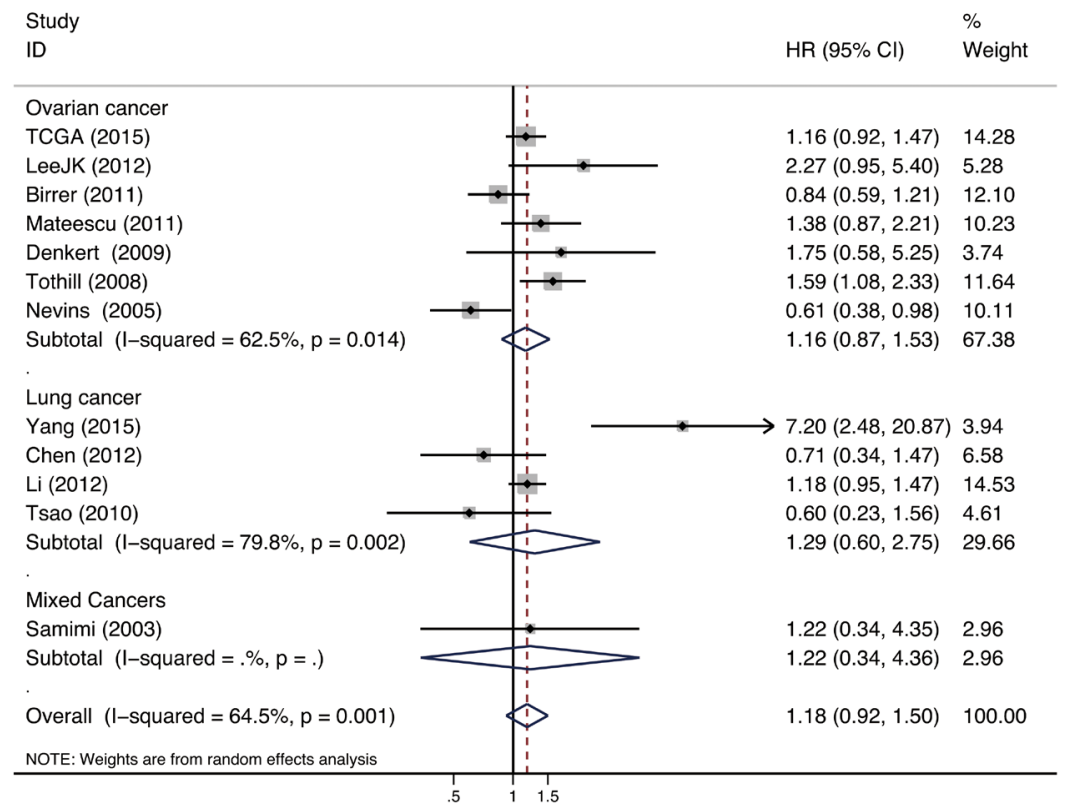

Figure 7: ATP7A and overall survival. Forest plot presents summary hazard ratios (HRs) and 95\% confidence intervals (CIs) of correlation between ATP7A expression and overall survival (OS) in epithelial cancers.

\section{ATP7A and Progression-free/Disease-free Survival}

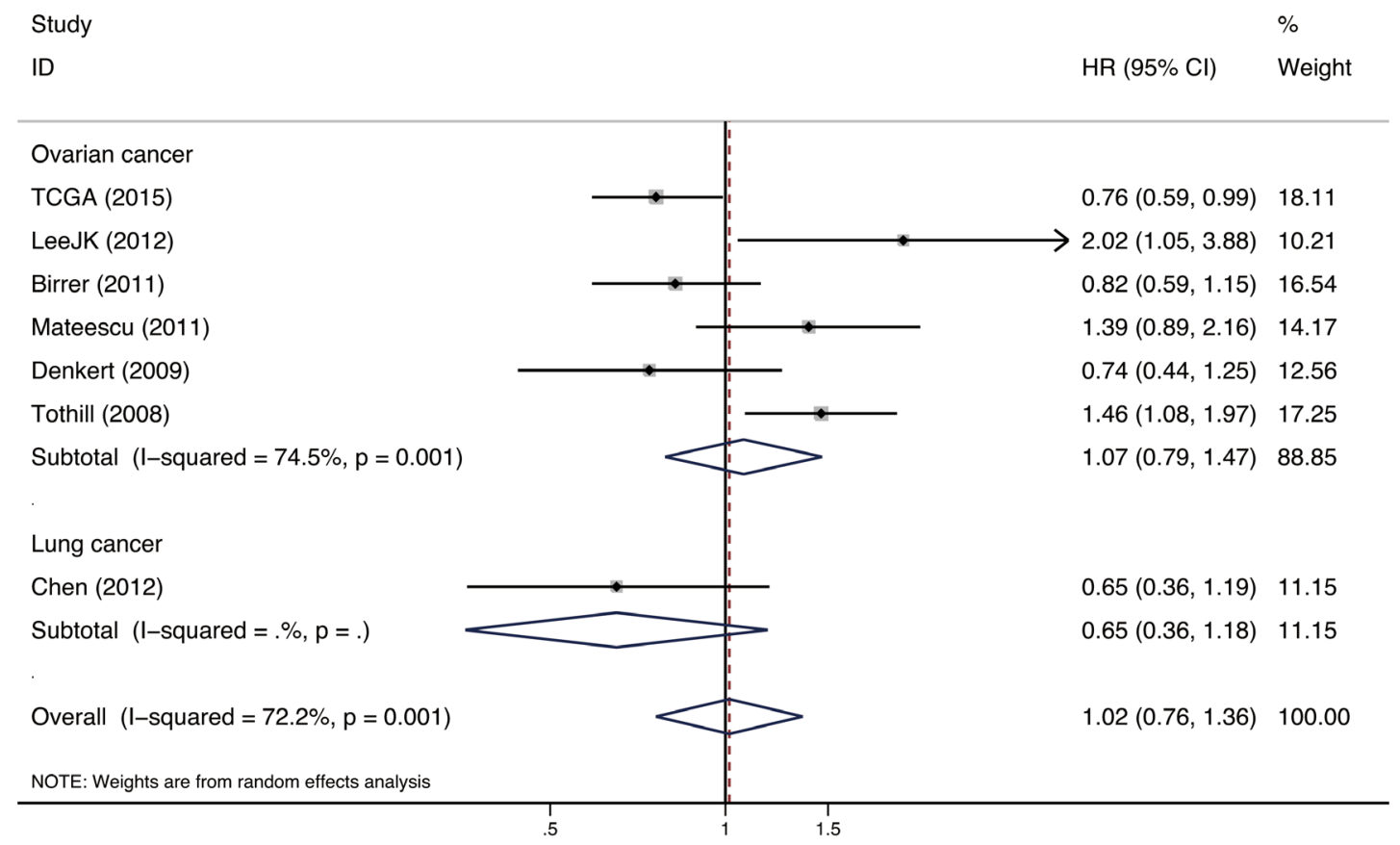

Figure 8: ATP7A and progression-free survival/disease-free survival. Forest plot presents summary hazard ratios (HRs) and 95\% confidence intervals (CIs) of correlation between ATP7A expression and progression free survival/disease-free survival (PFS/DFS in epithelial cancers. 
Table 3: Subgroup analyses of ATP7A and ATP7B for survival.

\begin{tabular}{|c|c|c|c|c|c|c|c|c|c|}
\hline \multirow{2}{*}{\multicolumn{2}{|c|}{ End-point }} & \multicolumn{4}{|c|}{ ATP7A } & \multicolumn{4}{|c|}{ ATP7B } \\
\hline & & $\mathbf{N}$ & HR (95\%CI) & $P$ & $I^{2}(\%)$ & $\mathbf{N}$ & HR (95\%CI) & $\begin{array}{l}P \\
(\%)\end{array}$ & $\overline{I^{2}}$ \\
\hline \multicolumn{10}{|l|}{ Cancer type } \\
\hline \multirow[t]{2}{*}{ Ovarian cancer } & OS & 7 & $1.16(0.87,1.53)$ & 0.306 & 62.5 & 10 & $1.07(0.83,1.40)$ & 0.594 & 57.8 \\
\hline & $\begin{array}{l}\text { PFS/ } \\
\text { DFS }\end{array}$ & 6 & $1.07(0.79,1.47)$ & 0.651 & 74.5 & 7 & $0.87(0.64,1.19)$ & 0.387 & 72.9 \\
\hline \multirow[t]{2}{*}{ Lung cancer } & OS & 4 & $1.29(0.60,2.75)$ & 0.514 & 79.8 & 3 & $0.83(0.38,1.81)$ & 0.633 & 60.0 \\
\hline & $\begin{array}{l}\text { PFS/ } \\
\text { DFS }\end{array}$ & 1 & $0.65(0.36,1.18)$ & 0.158 & - & 1 & $0.97(0.50,1.88)$ & 0.928 & - \\
\hline \multirow{2}{*}{$\begin{array}{l}\text { Endometrial } \\
\text { cancer }\end{array}$} & OS & - & - & - & - & 1 & $8.26(0.91,74.92)$ & 0.061 & - \\
\hline & $\begin{array}{l}\text { PFS/ } \\
\text { DFS }\end{array}$ & - & - & - & - & 1 & $3.46(2.07,5.79)$ & 0.022 & - \\
\hline \multirow[t]{2}{*}{$\begin{array}{l}\text { Colorectal } \\
\text { cancer }\end{array}$} & OS & - & - & - & - & - & - & - & - \\
\hline & $\begin{array}{l}\text { PFS/ } \\
\text { DFS }\end{array}$ & - & - & - & - & 1 & $8.28(1.36,50.46)$ & 0.000 & - \\
\hline \multirow[t]{2}{*}{ Mixed cancers } & OS & 1 & $1.22(0.34,4.36)$ & 0.188 & - & - & - & - & - \\
\hline & $\begin{array}{l}\text { PFS/ } \\
\text { DFS }\end{array}$ & - & - & - & - & - & - & - & - \\
\hline \multicolumn{10}{|c|}{ Chemotherapy regimen } \\
\hline \multirow[t]{2}{*}{ Platinum-based } & OS & 12 & $1.16(0.92,1.48)$ & 0.170 & 62.2 & 14 & $1.06(0.82,1.37)$ & 0.650 & 57.1 \\
\hline & $\begin{array}{l}\text { PFS/ } \\
\text { DFS }\end{array}$ & 7 & $1.04(0.77,1.39)$ & 0.754 & 72.1 & 11 & $1.23(0.86,1.75)$ & 0.255 & 81.4 \\
\hline \multicolumn{10}{|l|}{ Geographic region } \\
\hline \multirow[t]{2}{*}{ America } & OS & 6 & $0.95(0.69,1.32)$ & 0.762 & 55.5 & 5 & $0.97(0.65,1.45)$ & 0.882 & 70.8 \\
\hline & $\begin{array}{l}\text { PFS/ } \\
\text { DFS }\end{array}$ & 3 & $0.97(0.64,1.49)$ & 0.902 & 73.3 & 3 & $0.92(0.48,1.79)$ & 0.812 & 89.3 \\
\hline \multirow[t]{2}{*}{ Asia } & OS & 3 & $1.63(0.63,4.18)$ & 0.313 & 84.4 & 6 & $1.54(0.92,2.59)$ & 0.103 & 50.4 \\
\hline & $\begin{array}{l}\text { PFS/ } \\
\text { DFS }\end{array}$ & 1 & $0.65(0.36,1.18)$ & 0.158 & - & 3 & $1.56(0.67,3.63)$ & 0.298 & 58.3 \\
\hline \multirow[t]{2}{*}{ Europe } & OS & 2 & $1.43(0.93,2.20)$ & 0.102 & 0.0 & 2 & $0.75(0.46,1.21)$ & 0.241 & 0.0 \\
\hline & $\begin{array}{l}\text { PFS/ } \\
\text { DFS }\end{array}$ & 2 & $1.03(0.56,1.91)$ & 0.925 & 69.3 & 3 & $1.49(0.61,3.65)$ & 0384 & 88.6 \\
\hline \multirow[t]{2}{*}{ Oceania } & OS & 1 & $1.59(1.08,2.34)$ & 0.018 & - & 1 & $0.86(0.59,1.26)$ & 0.436 & - \\
\hline & $\begin{array}{l}\text { PFS/ } \\
\text { DFS }\end{array}$ & 1 & $1.46(1.08,1.97)$ & 0.014 & - & 1 & $0.84(0.61,1.16)$ & 0.288 & - \\
\hline \multicolumn{10}{|l|}{ Data origin } \\
\hline \multirow[t]{2}{*}{ Dataset } & OS & 8 & $1.11(0.85,1.46)$ & 0.444 & 60.3 & 8 & $0.91(0.70,1.18)$ & 0.491 & 53.1 \\
\hline & $\begin{array}{l}\text { PFS/ } \\
\text { DFS }\end{array}$ & 6 & $1.07(0.79,1.47)$ & 0.651 & 74.5 & 6 & $0.83(0.60,1.16)$ & 0.278 & 75.8 \\
\hline \multirow[t]{2}{*}{ Literature } & OS & 4 & $1.51(0.71,3.24)$ & 0.285 & 76.7 & 6 & $1.54(0.92,2.59)$ & 0.103 & 50.4 \\
\hline & $\begin{array}{l}\text { PFS/ } \\
\text { DFS }\end{array}$ & 1 & $0.65(0.36,1.18)$ & 0.158 & - & 5 & $2.27(1.16,4.42)$ & 0.016 & 69.0 \\
\hline \multicolumn{10}{|l|}{ Detection method } \\
\hline \multirow[t]{2}{*}{ Gene array } & OS & 8 & $1.11(0.85,1.46)$ & 0.444 & 60.3 & 8 & $0.91(0.70,1.18)$ & 0.491 & 53.1 \\
\hline & $\begin{array}{l}\text { PFS/ } \\
\text { DFS }\end{array}$ & 6 & $1.07(0.79,1.47)$ & 0.651 & 74.5 & 6 & $0.83(0.60,1.16)$ & 0.278 & 75.8 \\
\hline \multirow[t]{2}{*}{ IHC } & OS & 4 & $1.51(0.71,3.24)$ & 0.285 & 76.7 & 5 & $1.69(0.86,3.30)$ & 0.126 & 58.8 \\
\hline & $\begin{array}{l}\text { PFS/ } \\
\text { DFS }\end{array}$ & 1 & $0.65(0.36,1.18)$ & 0.158 & - & 4 & $2.19(0.92,5.22)$ & 0.075 & 74.9 \\
\hline \multirow[t]{2}{*}{ qRT-PCR } & OS & - & - & - & - & 1 & $1.21(0.61,2.41)$ & 0.587 & - \\
\hline & $\begin{array}{l}\text { PFS/ } \\
\text { DFS }\end{array}$ & - & - & - & - & 1 & $2.81(1.36,5.81)$ & 0.005 & - \\
\hline
\end{tabular}

P-value for significance of HR. (P < 0.050) HR: hazard ratio; CI: confidence interval ; IHC: immunochemistry; qRT-PCR: real-time polymerase chain reaction 
Table 4: Meta-analysis and subgroups analyses of treatment response.

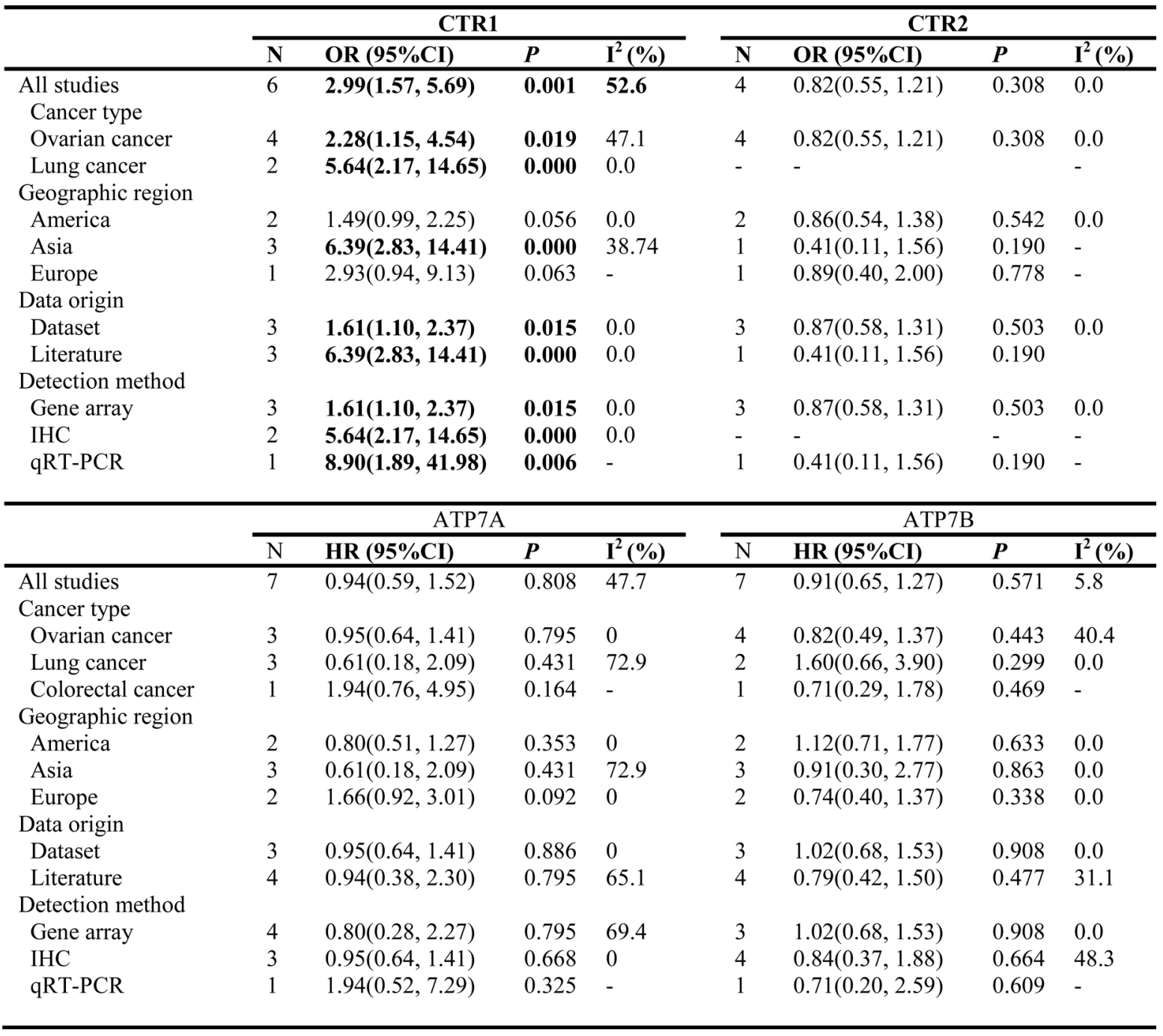

$P$-value for significance of OR. $(\mathrm{P}<0.050) \mathrm{HR}$ : hazard ratio; $\mathrm{CI}$ : confidence interval ; IHC: immunochemistry; qRT-PCR: real-time polymerase chain reaction

\section{Sensitivity analysis and Publication bias}

According to sensitivity analyses, the summary HRs for OS of CTR1 and PFS of CTR2 were influenced by one study in the "Europe" subgroup [32]. All the remaining studies did not influence the summary HRs and ORs. No evidence of publication bias was found in overall metaanalyses using funnel plots and Egger's test (all $P>0.05$ ).

\section{DISCUSSION}

In this meta-analysis, we systematically evaluated the relationship between four copper transporters (CTR1, CTR2, ATP7A and ATP7B) that influence cellular platinum accumulation and prognosis of cancer patients who received chemotherapy. Our results showed that high CTR1 expression was significantly associated with a favorable OS, PFS, DFS and TR, suggesting that CTR1 is a potential prognostic factor for survival in cancer patients underwent chemotherapy and a treatment target for overcoming platinum resistance.

Ever since the introduction of chemotherapy, drug response becomes a major determiner of cancer prognosis, which parallels with FIGO stage and residual debulked tumor volume. The cure rate of testicular cancer zoomed from $15 \%$ to $90 \%$ attributing to a great response to platinum-based chemotherapy[36]. In ovarian cancer patients, different histologic subtypes are characterized by distinct platinum response leading to different prognosis, the better the response the better the survival. Switching on and off of an array of genes such as BRCA1, BRCA2, Akt1, ERCC1 and ABCB1 were identified to modulate sensitivity toward chemotherapy in cancer patients. We demonstrated that CTR1 is one of those genes whose expression is significantly associated with OS, PFS, DFS and chemotherapy response in patients underwent chemotherapy, especially in those who received platinum- 


\section{ATP7B and Overall Survival}

\begin{tabular}{|c|c|c|}
\hline $\begin{array}{l}\text { Study } \\
\text { ID }\end{array}$ & $\mathrm{HR}(95 \% \mathrm{Cl})$ & $\begin{array}{l}\% \\
\text { Weight }\end{array}$ \\
\hline \multicolumn{3}{|l|}{ Ovarian cancer } \\
\hline TCGA (2015) & $0.82(0.63,1.07)$ & 12.78 \\
\hline Katagiri (2013) & $1.93(0.81,4.63)$ & 5.37 \\
\hline LeeJK (2012) & $1.42(0.70,2.90)$ & 6.84 \\
\hline Birrer (2011) & $1.65(1.13,2.41)$ & 11.15 \\
\hline Mateescu (2011) & $0.78(0.45,1.35)$ & 8.74 \\
\hline Denkert (2009) & $0.65(0.23,1.78)$ & 4.33 \\
\hline Tothill (2008) & $0.86(0.59,1.26)$ & 11.14 \\
\hline Nevins (2005) & $0.68(0.41,1.14)$ & 9.24 \\
\hline Nakayama (2004) & $2.55(1.10,5.91)$ & 5.62 \\
\hline Nakayama (2002) & $1.21(0.61,2.41)$ & 7.09 \\
\hline Subtotal $(I-$ squared $=57.8 \%, p=0.011)$ & $1.07(0.83,1.40)$ & 82.29 \\
\hline \multicolumn{3}{|l|}{ Lung cancer } \\
\hline Yang (2015) & $1.78(0.78,4.03)$ & 5.79 \\
\hline Chen (2012) & $0.58(0.26,1.29)$ & 5.97 \\
\hline Tsao (2010) & $0.52(0.20,1.36)$ & 4.74 \\
\hline Subtotal $(I-$ squared $=60.0 \%, p=0.082$ ) & $0.83(0.38,1.81)$ & 16.50 \\
\hline \multicolumn{3}{|l|}{ Endometrial cancer } \\
\hline \multicolumn{3}{|l|}{ Aida $(2005)$} \\
\hline \multirow{2}{*}{\multicolumn{3}{|c|}{ Subtotal $(I-$ squared $=. \%, p=)}}$. \\
\hline & & \\
\hline Overall $(I-$ squared $=57.1 \%, p=0.004)$ & $1.06(0.82,1.37)$ & 100.00 \\
\hline NOTE: Weights are from random effects analysis & & \\
\hline
\end{tabular}

Figure 9: ATP7B and overall survival. Forest plot presents summary hazard ratios (HRs) and 95\% confidence intervals (CIs) of correlation between ATP7B expression and overall survival (OS) in epithelial cancers.

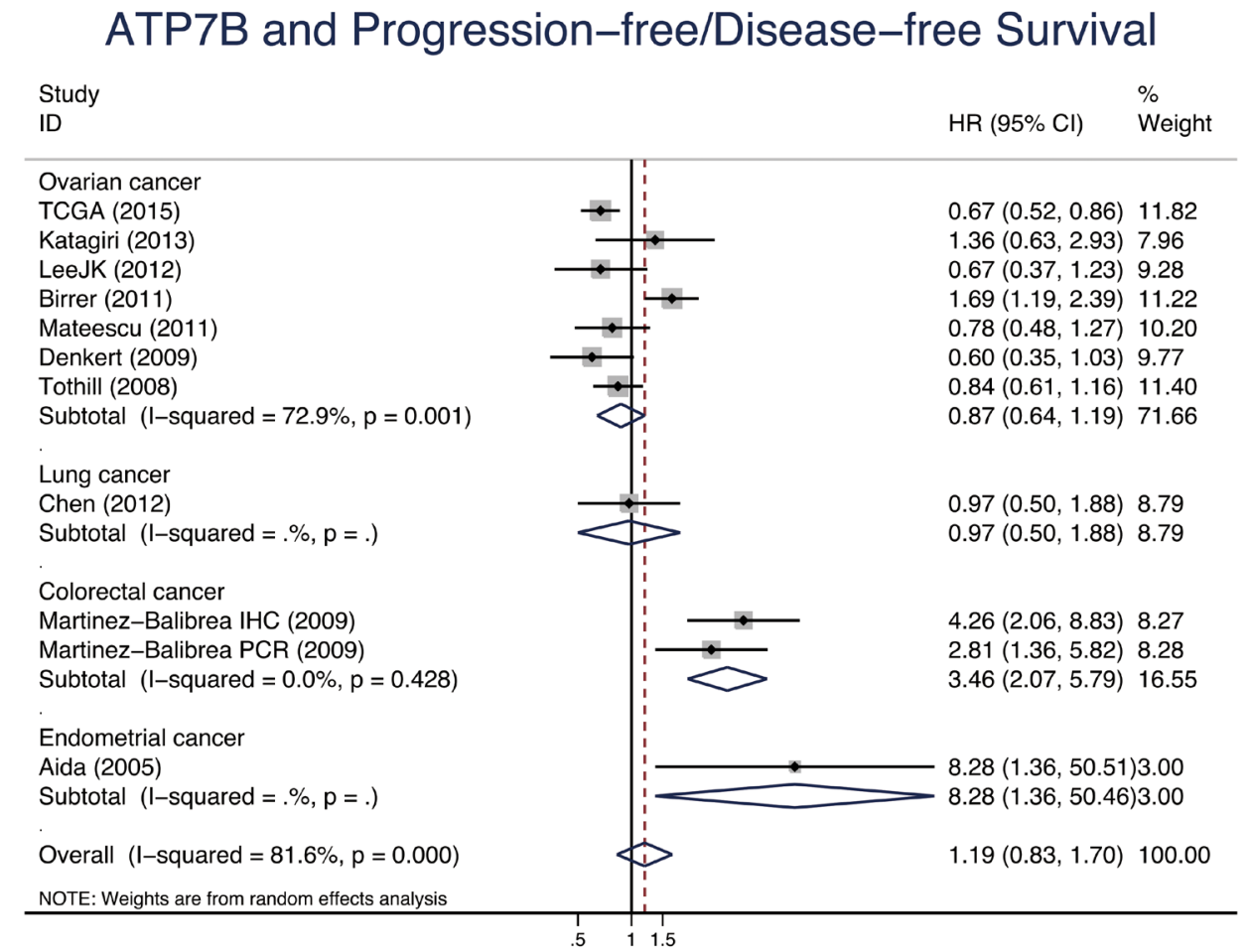

Figure 10: ATP7B and progression-free survival/disease-free survival. Forest plot presents summary hazard ratios (HRs) and $95 \%$ confidence intervals (CIs) of correlation between ATP7B expression and progression free survival/disease-free survival (PFS/DFS in epithelial cancers. 
based chemotherapy. CTR1 contributes to the active cellular uptake of cisplatin, carboplatin and oxaliplatin. In our study, most patients received cisplatin- or carboplatinbased chemotherapy, both of which are important substrates of hCTR1. It was reported that hCTR1 was responsible to a $60 \%$ to $70 \%$ cellular uptake of cisplatin and a $30 \%$ to $40 \%$ uptake of carboplatin $[37,38]$. So far, no other chemotherapy agents were identified as CTR1 substrates. Unfortunately, we were not able to analyze the impact of CTR1 on patients underwent non-platinum containing chemotherapy due to insufficient data. We found a significant association between CTR1 expression and tumor prognosis by pooling data from 5 studies and 8 datasets involving 2149 patients. Although all data were retrospectively obtained, multiple data sources greatly decreased the publication bias and efficiently increased the validity of the results. Significant clinical associations of CTR1 expression were constant between all subgroups: cancer type, geographical region, data origin and detection method with acceptable heterogeneities.

In our study, we focused on evaluating the initial copper transporter expression in blood and tissue specimens obtained before or during primary surgery. We found that a high initial hCTR1 expression predicted improved prognosis in ovarian and lung cancer patients. However, pre-clinical evidence indicates that the expression of CTR1 is dynamic. Prompt reaction of CTR1 to acute copper concentration alteration is a wellrecognized mechanism that regulates hCTR1 expression. Excessive copper triggers rapid hCTR1 internalization by endocytosis, which reduces the transportation activity of CTR1 [39, 40]. Copper deficiency up-regulates transcription-factor-specific protein 1 (Sp1), which binds to hCTR1 promoter region and enhances hCTR1 expression[41]. Some studies also reported that cisplatin induces rapid hCTR1 degradation through ubiquitinmediated proteolysis [42, 43]. According to Jandial et al., administration of proteasome inhibitor bortezomib successfully blocked platinum-induced CTR1 degradation and increased platinum accumulation in peritoneal tumors [44]. Whether hCTR1 expression is subjected to similar dynamic change induced by substrate stimulation in cancer patients and whether this change is associated with tumor prognosis are still undetermined.

In relation to chemotherapy response and cancer prognosis, according to our results, CTR1 is of particular attractiveness to serve as a promising target to circumvent platinum resistance. Since correlation between reduced hCTR1 expression and platinum resistance was exhibited in precedent and the present studies, combination of copper lowering agents and platinum based chemotherapy, which stimulates hCTR1 expression and improve platinum uptake, is a promising strategy to treat hCTR1 deficiency mediated platinum resistance. Ishida $\mathrm{S}$ et al. successfully enhanced the cytotoxic effect of cisplatin on human cervical and ovarian cancer cells by up-regulating hCTR 1 expression through administration of a copper chelating agent tetrathiomolybdate in vitro [45]. Fu et al. carried out the first clinical trial to explore the clinical value of copper chelating agents. Approximate $20 \%$ of the platinum resistant patients responded to platinum after receiving copper chelator [46]. However, this strategy was not tested in clinical trials.

We initially expected diverse prognostic values of CTR2 in distinct cancer types, in patients with different genetic background and in studies applied inconsistent CTR2 detection methods based on existing studies. Though CTR2 shares a substantial similar structure with CTR1, it plays drastically different role in platinum uptake. Unlike CTR1, preclinical studies revealed that high CTR2 level was associated with decreased cellular platinum uptake and poor drug response in ovarian cancer $[8,47]$. Additionally, copper modulation correlated CTR2 with angiogenesis in clear cell renal cell carcinoma, where lower CTR2 expression predicted shorter OS and DFS [48]. Heterogeneity of genetic background of patients not only influences basic CTR2 level of the population but also alters various gene levels and pathways that CTR2 interacts with. Moreover, CTR2 regulates cellular platinum uptake through a protein-protein interaction with CTR1 [8], the relationship between CTR2 and platinum sensitivity is better revealed post-transcriptionally. When evaluating the relationship of CTR2 and cancer prognosis in a clinical setting, we did not find any evidence for correlation between CTR2 and prognosis in different cancer types, data origins and detection methods but saw a discrepancy in geographic stratification of the America and the Europe subgroups regarding PFS. We found that the detection method, cancer type, methodology, type of samples were consistent in all 5 studies from these two subgroups. One study from the Europe subgroup reported a specific result that was opposite to all other studies [32]. We did not have enough evidence to determine whether the inconsistent result was due to genetic difference or simply small size effect. We believed it was necessary to investigate the issue in a much greater clinical scale and covered as much as detection methods as possible since our results were only obtained on the basis of a series gene-array analyses due to limited data, which might not necessarily exhibit the correlation between CTR2 protein expression and platinum resistance.

The present study is the first meta-analysis regarding the impact of copper transporters on the outcomes of cancer patients who received chemotherapy to our knowledge. We used multiple data sources and performed subgroup analyses stratified by geological regions of publication, data origins and detection methods to accommodate possible biases and provide detailed results that help accelerate the relevant clinical translation. However, all the enrolled studies are retrospective, which provide evidence of Level II-1 or II-2 [49] and limit the reliability of our findings. The relationship between CTR1 
and chemotherapy clinical outcomes in tumor patients needs validation by large-scale randomized controlled trials. In addition, most data for meta-analysis were obtained from studies on ovarian cancer and lung cancer, which restricts the application of the conclusions in other malignancies.

To sum up, high CTR1 level is associated with improved survival and TR in cancer patients, especially in patients with ovarian or lung cancer undergoing platinumbased chemotherapy, Prospective randomized studies are needed to verify the clinical value of CTR 1 in tumor patients.

\section{MATERIALS AND METHODS}

\section{Search strategy}

An independent systematic literature search of studies in all languages to May 2016 across PubMed, EMBASE, and Chinese Wanfang databases was conducted. The search terms used were "copper transporter 1", "human copper transporter 1", "copper transporter 2", "ATP7A", "ATP7B" and "platinum-based chemotherapy". The electronic search of literature was supplemented by reviewing the reference lists of retrieved studies. Additionally we searched the Gene Expression Omnibus (GEO) (http://www.ncbi.nlm.nih.gov/gds) and the Cancer Genome Atlas (TCGA) datasets (http://cancergenome.nih. gov) for relevant information. The key words used were "CTR1", “CTR2”, “ATP7A", “ATP7B”, "platinum” and "Homo sapiens".

\section{Study Selection}

A two-step study selection was conducted including initial review of titles and abstracts, and a second review of full texts retrieved. The inclusion criteria for identifying eligible studies included: 1) clinical studies measured CTR1, CTR2, ATP7A or ATP7B in solid tumors regardless of detection methods; 2) published prospective and retrospective cohort studies; 3) studies provided information of survival and chemotherapy response. Exclusion criteria included: 1) the patients enrolled did not receive chemotherapy; 2) studies focused on gene polymorphisms or DNA methylation; 3) studies investigated different end-points; 4) studies did not provide sufficient data. Eligible datasets should meet the following criteria: 1) cancer patients received chemotherapy; 2) the sample size should be more than 40;3) gene array was performed using Affymetrix platform. If multiple studies shared overlapped populations, the study with the largest sample size was included. If multiple studies with overlapped populations used different detection methods, all were included.

\section{Quality assessment and Data extraction}

The quality of the included literatures was assessed according to the Newcastle-Ottawa Scale (NOS) for cohort studies[50]. We extracted the following data from each individual eligible study or dataset: first author's/ contributor's name, year of publication, country, sample size, number of cases and controls, tumor type, detection methods and detection results. Two authors extracted the data independently and disagreements were solved by discussion.

\section{Statistical Methods}

The aim of our study was to investigate the relationship between the expression of copper transporters and the prognosis of cancer patients who received chemotherapy. The primary outcome measure was overall survival (OS), and secondary measures were progressionfree survival (PFS)/disease-free survival (DFS) and treatment response (TR). The impact of platinumtransporter expression on OS, PFS and DFS was assessed by hazard ratio (HR), odds ratio (OR) in case of TR, and its 95\% confidence interval (CI). The HRs and 95\% CIs were extracted from eligible studies directly or calculated using the survival data extracted from the reported Kaplan-Meier curves with the software GetDATA Graph Digitizer 2.25 according to the method described by Tierney et al. [51]. An on-line survival analysis software KM-plotter (http:// kmplot.com/analysis/) with the selection of optimal cutoff point and microarray probe set was used to calculate the HRs and 95\% CIs using data from GEO and TCGA datasets [52-54]. Random-effects models were employed to pool the HRs, ORs and 95\%CIs [55]. The heterogeneity across studies was assessed according to $I^{2}$ statistic with the method of Higgins et al. [56]. A heterogeneity was considered significant when $I^{2} \geq 50 \%$. Subgroup analyses stratified by tumor type, geographic region, data origin and detection method were performed. Sensitivity analyses were performed by excluding one study at a time to evaluate the influence of single studies on summary HRs. Publication bias was assessed by funnel plot and Egger's test [57]. All analyses were conducted using STATA14 (MP-Parallel Edition, College Station, Texas 77845 USA). $P$ values less than 0.05 were considered statistically significant.

\section{Abbreviations}

HR, hazard ratio; CI, confidence interval; CTR1, copper transporter 1; hCTR1, human copper transporter 1; CTR2, copper transporter 2; ATP7A, Copper-transporting P-type adenosine triphosphatase 1; ATP7B, coppertransporting $\mathrm{P}$-type adenosine triphosphatase 2 . 


\section{ACKNOWLEDGEMENTS}

We thanked Xiaoqing Yi for her support of data extraction and Qiang Yang for his advice on manuscript preparation.

\section{CONFLICTS OF INTEREST}

There is no conflict of interest regarding this study.

\section{FUNDING}

This work was supported by National Natural Science Foundation of China $(81272860,81472443$, 81572572).

\section{Author contributions}

Si Sun and Zehua Wang designed the study. Jing Cai reviewed the methodology of the study. Si Sun and Simei Zhao screened the studies and extracted data. Qiang Yang and Si Sun conducted statistical analysis. Jing Cai and Zehua Wang reviewed the reliability of the results. Si Sun and Jing Cai prepared the manuscript and figures. All authors reviewed and approved the final version of the paper.

\section{REFERENCES}

1. Ho GY, Woodward N and Coward JI. Cisplatin versus carboplatin: comparative review of therapeutic management in solid malignancies. Critical reviews in oncology/ hematology. 2016.

2. Kim ES, Tang X, Peterson DR, Kilari D, Chow CW, Fujimoto J, Kalhor N, Swisher SG, Stewart DJ, Wistuba, II and Siddik ZH. Copper transporter CTR1 expression and tissue platinum concentration in non-small cell lung cancer. Lung cancer. 2014; 85(1):88-93.

3. Matsumoto S, Tanaka T, Kurokawa H, Matsuno K, Hayashida $\mathrm{Y}$ and Takahashi T. Effect of copper and role of the copper transporters ATP7A and CTR1 in intracellular accumulation of cisplatin. Anticancer research. 2007; 27(4B):2209-2216.

4. Gately DP and Howell SB. Cellular accumulation of the anticancer agent cisplatin: a review. British journal of cancer. 1993; 67(6):1171-1176.

5. Hall MD, Okabe M, Shen DW, Liang XJ and Gottesman MM. The role of cellular accumulation in determining sensitivity to platinum-based chemotherapy. Annual review of pharmacology and toxicology. 2008; 48:495-535.

6. Katano K, Kondo A, Safaei R, Holzer A, Samimi G, Mishima M, Kuo YM, Rochdi M and Howell SB. Acquisition of resistance to cisplatin is accompanied by changes in the cellular pharmacology of copper. Cancer research. 2002; 62(22):6559-6565.

7. Safaei R, Katano K, Samimi G, Naerdemann W, Stevenson JL, Rochdi M and Howell SB. Cross-resistance to cisplatin in cells with acquired resistance to copper. Cancer chemotherapy and pharmacology. 2004; 53(3):239-246.

8. Ohrvik H, Nose Y, Wood LK, Kim BE, Gleber SC, Ralle $\mathrm{M}$ and Thiele DJ. Ctr2 regulates biogenesis of a cleaved form of mammalian Ctr1 metal transporter lacking the copper- and cisplatin-binding ecto-domain. Proceedings of the National Academy of Sciences of the United States of America. 2013; 110(46):E4279-4288.

9. Yoshizawa K, Nozaki S, Kitahara H, Ohara T, Kato K, Kawashiri S and Yamamoto E. Copper efflux transporter (ATP7B) contributes to the acquisition of cisplatinresistance in human oral squamous cell lines. Oncology reports. 2007; 18(4):987-991.

10. Larson CA, Blair BG, Safaei R and Howell SB. The role of the mammalian copper transporter 1 in the cellular accumulation of platinum-based drugs. Molecular pharmacology. 2009; 75(2):324-330.

11. Blair BG, Larson CA, Safaei R and Howell SB. Copper transporter 2 regulates the cellular accumulation and cytotoxicity of Cisplatin and Carboplatin. Clin Cancer Res. 2009; 15(13):4312-4321.

12. Samimi G, Katano K, Holzer AK, Safaei R and Howell SB Modulation of the cellular pharmacology of cisplatin and its analogs by the copper exporters ATP7A and ATP7B. Molecular pharmacology. 2004; 66(1):25-32.

13. Howell SB, Safaei R, Larson CA and Sailor MJ. Copper transporters and the cellular pharmacology of the platinumcontaining cancer drugs. Molecular pharmacology. 2010; 77(6):887-894.

14. Safaei R, Adams PL, Mathews RA, Manorek G and Howell SB. The role of metal binding and phosphorylation domains in the regulation of cisplatin-induced trafficking of ATP7B. Metallomics. 2013; 5(8):964-972.

15. Tadini-Buoninsegni F, Bartolommei G, Moncelli MR, Inesi G, Galliani A, Sinisi M, Losacco M, Natile G and Arnesano F. Translocation of platinum anticancer drugs by human copper ATPases ATP7A and ATP7B. Angewandte Chemie. 2014; 53(5):1297-1301.

16. Yang $\mathrm{T}$, Chen $\mathrm{M}$, Chen $\mathrm{T}$ and Thakur A. Expression of the copper transporters hCtr1, ATP7A and ATP7B is associated with the response to chemotherapy and survival time in patients with resected non-small cell lung cancer. Oncology letters. 2015; 10(4):2584-2590.

17. Hsu KF, Shen MR, Huang YF, Cheng YM, Lin SH, Chow $\mathrm{NH}$, Cheng SW, Chou CY and Ho CL. Overexpression of the RNA-binding proteins Lin28B and IGF2BP3 (IMP3) is associated with chemoresistance and poor disease outcome in ovarian cancer. British journal of cancer. 2015; 113(3):414-424.

18. Ogane N, Yasuda M, Kameda Y, Yokose T, Kato H, Itoh A, Nishino S, Hashimoto Y and Kamoshida S. Prognostic 
value of organic anion transporting polypeptide 1B3 and copper transporter 1 expression in endometrial cancer patients treated with paclitaxel and carboplatin. Biomedical research. 2013; 34(3):143-151.

19. Katagiri H, Nakayama K, Rahman MT, Rahman M, Katagiri A, Ishibashi T, Ishikawa M, Iida K, Nakayama S, Otsuki Y and Miyazaki K. Is ATP7B a predictive marker in patients with ovarian carcinoma treated with platinumtaxane combination chemotherapy? International journal of gynecological cancer. 2013; 23(1):60-64.

20. Li ZH, Qiu MZ, Zeng ZL, Luo HY, Wu WJ, Wang F, Wang ZQ, Zhang DS, Li YH and Xu RH. Copper-transporting P-type adenosine triphosphatase (ATP7A) is associated with platinum-resistance in non-small cell lung cancer (NSCLC). Journal of translational medicine. 2012; 10:21.

21. Chen HH, Yan JJ, Chen WC, Kuo MT, Lai YH, Lai WW, Liu HS and $\mathrm{Su}$ WC. Predictive and prognostic value of human copper transporter 1 (hCtr1) in patients with stage III non-small-cell lung cancer receiving first-line platinum-based doublet chemotherapy. Lung cancer. 2012; 75(2):228-234.

22. Lee YY, Choi CH, Do IG, Song SY, Lee W, Park HS, Song TJ, Kim MK, Kim TJ, Lee JW, Bae DS and Kim BG. Prognostic value of the copper transporters, CTR1 and CTR2, in patients with ovarian carcinoma receiving platinum-based chemotherapy. Gynecologic oncology. 2011; 122(2):361-365.

23. Martinez-Balibrea E, Martinez-Cardus A, Musulen E, Gines A, Manzano JL, Aranda E, Plasencia C, Neamati N and Abad A. Increased levels of copper efflux transporter ATP7B are associated with poor outcome in colorectal cancer patients receiving oxaliplatin-based chemotherapy. International journal of cancer. 2009; 124(12):2905-2910.

24. Aida T, Takebayashi Y, Shimizu T, Okamura C, Higasimoto M, Kanzaki A, Nakayama K, Terada K, Sugiyama T, Miyazaki K, Ito K, Takenoshita S and Yaegashi N. Expression of copper-transporting P-type adenosine triphosphatase (ATP7B) as a prognostic factor in human endometrial carcinoma. Gynecologic oncology. 2005; 97(1):41-45.

25. Nakayama K, Kanzaki A, Terada K, Mutoh M, Ogawa K, Sugiyama T, Takenoshita S, Itoh K, Yaegashi N, Miyazaki $\mathrm{K}$, Neamati N and Takebayashi Y. Prognostic value of the $\mathrm{Cu}$-transporting ATPase in ovarian carcinoma patients receiving cisplatin-based chemotherapy. Clin Cancer Res. 2004; 10(8):2804-2811.

26. Samimi G, Varki NM, Wilczynski S, Safaei R, Alberts DS and Howell SB. Increase in expression of the copper transporter ATP7A during platinum drug-based treatment is associated with poor survival in ovarian cancer patients. Clinical cancer research. 2003; 9(16 Pt 1):5853-5859.

27. Nakayama K, Kanzaki A, Ogawa K, Miyazaki K, Neamati $\mathrm{N}$ and Takebayashi Y. Copper-transporting P-type adenosine triphosphatase (ATP7B) as a cisplatin based chemoresistance marker in ovarian carcinoma: comparative analysis with expression of MDR1, MRP1, MRP2, LRP and BCRP. International journal of cancer. 2002; 101(5):488495.

28. Martin JW, Chilton-MacNeill S, Koti M, van Wijnen AJ, Squire JA and Zielenska M. Digital expression profiling identifies RUNX2, CDC5L, MDM2, RECQL4, and CDK4 as potential predictive biomarkers for neo-adjuvant chemotherapy response in paediatric osteosarcoma. PloS one. 2014; 9(5):e95843.

29. Ferriss JS, Kim Y, Duska L, Birrer M, Levine DA, Moskaluk C, Theodorescu D and Lee JK. Multi-gene expression predictors of single drug responses to adjuvant chemotherapy in ovarian carcinoma: predicting platinum resistance. PloS one. 2012; 7(2):e30550.

30. Mateescu B, Batista L, Cardon M, Gruosso T, de Feraudy Y, Mariani O, Nicolas A, Meyniel JP, Cottu P, SastreGarau X and Mechta-Grigoriou F. miR-141 and miR-200a act on ovarian tumorigenesis by controlling oxidative stress response. Nature medicine. 2011; 17(12):1627-1635.

31. Zhu CQ, Ding K, Strumpf D, Weir BA, Meyerson M, Pennell N, Thomas RK, Naoki K, Ladd-Acosta C, Liu N, Pintilie M, Der S, Seymour L, Jurisica I, Shepherd FA and Tsao MS. Prognostic and predictive gene signature for adjuvant chemotherapy in resected non-small-cell lung cancer. Journal of clinical oncology. 2010; 28(29):44174424.

32. Denkert C, Budczies J, Darb-Esfahani S, Gyorffy B, Sehouli J, Konsgen D, Zeillinger R, Weichert W, Noske A, Buckendahl AC, Muller BM, Dietel M and Lage H. A prognostic gene expression index in ovarian cancer - validation across different independent data sets. The Journal of pathology. 2009; 218(2):273-280.

33. Tothill RW, Tinker AV, George J, Brown R, Fox SB, Lade S, Johnson DS, Trivett MK, Etemadmoghadam D, Locandro B, Traficante N, Fereday S, Hung JA, Chiew YE, Haviv I, Australian Ovarian Cancer Study G, et al. Novel molecular subtypes of serous and endometrioid ovarian cancer linked to clinical outcome. Clin Cancer Res. 2008; 14(16):5198-5208.

34. Bonome T, Levine DA, Shih J, Randonovich M, PiseMasison CA, Bogomolniy F, Ozbun L, Brady J, Barrett JC, Boyd J and Birrer MJ. A gene signature predicting for survival in suboptimally debulked patients with ovarian cancer. Cancer research. 2008; 68(13):5478-5486.

35. Bild AH, Yao G, Chang JT, Wang Q, Potti A, Chasse D, Joshi MB, Harpole D, Lancaster JM, Berchuck A, Olson JA, Jr., Marks JR, Dressman HK, West M and Nevins JR. Oncogenic pathway signatures in human cancers as a guide to targeted therapies. Nature. 2006; 439(7074):353-357.

36. Jones RH and Vasey PA. Part I: testicular cancer-management of early disease. The Lancet Oncology. 2003; 4(12):730-737.

37. Holzer AK, Manorek GH and Howell SB. Contribution of the major copper influx transporter CTR1 to the cellular accumulation of cisplatin, carboplatin, and oxaliplatin. 
Molecular pharmacology. 2006; 70(4):1390-1394.

38. Lee J, Petris MJ and Thiele DJ. Characterization of mouse embryonic cells deficient in the ctr1 high affinity copper transporter. Identification of a Ctr1-independent copper transport system. The Journal of biological chemistry. 2002; 277(43):40253-40259.

39. Guo Y, Smith K, Lee J, Thiele DJ and Petris MJ. Identification of methionine-rich clusters that regulate copper-stimulated endocytosis of the human Ctr1 copper transporter. The Journal of biological chemistry. 2004; 279(17):17428-17433.

40. Molloy SA and Kaplan JH. Copper-dependent recycling of hCTR1, the human high affinity copper transporter. The Journal of biological chemistry. 2009; 284(43):2970429713.

41. Kuo MT, Fu S, Savaraj N and Chen HH. Role of the human high-affinity copper transporter in copper homeostasis regulation and cisplatin sensitivity in cancer chemotherapy. Cancer research. 2012; 72(18):4616-4621.

42. Holzer AK, Katano K, Klomp LW and Howell SB. Cisplatin rapidly down-regulates its own influx transporter hCTR1 in cultured human ovarian carcinoma cells. Clinical cancer research. 2004; 10(19):6744-6749.

43. Safaei R, Maktabi MH, Blair BG, Larson CA and Howell SB. Effects of the loss of Atox 1 on the cellular pharmacology of cisplatin. Journal of inorganic biochemistry. 2009; 103(3):333-341.

44. Jandial DD, Farshchi-Heydari S, Larson CA, Elliott GI, Wrasidlo WJ and Howell SB. Enhanced delivery of cisplatin to intraperitoneal ovarian carcinomas mediated by the effects of bortezomib on the human copper transporter 1. Clinical cancer research. 2009; 15(2):553-560.

45. Ishida S, McCormick F, Smith-McCune K and Hanahan D. Enhancing tumor-specific uptake of the anticancer drug cisplatin with a copper chelator. Cancer cell. 2010; 17(6):574-583.

46. Fu S, Hou MM, Wheler J, Hong D, Naing A, Tsimberidou A, Janku F, Zinner R, Piha-Paul S, Falchook G, Kuo MT and Kurzrock R. Exploratory study of carboplatin plus the copper-lowering agent trientine in patients with advanced malignancies. Investigational new drugs. 2014; 32(3):465472 .
47. Huang CP, Fofana M, Chan J, Chang CJ and Howell SB. Copper transporter 2 regulates intracellular copper and sensitivity to cisplatin. Metallomics. 2014; 6(3):654-661.

48. Xia Y, Liu L, Long Q, Bai Q, Wang J, Xu J and Guo J. Decreased expression of CTR2 predicts poor prognosis of patients with clear cell renal cell carcinoma. Urologic oncology. 2016; 34(1):5 e1-9.

49. U.S. Preventive Services Task Force. Guide to clinical preventive services: report of the U.S. Preventive Services Task Force. 1989; DIANE Publishing. pp. 24-. ISBN 9781-56806-297-6.

50. Stang A. Critical evaluation of the Newcastle-Ottawa scale for the assessment of the quality of nonrandomized studies in meta-analyses. European journal of epidemiology. 2010; 25(9):603-605.

51. Tierney JF, Stewart LA, Ghersi D, Burdett S and Sydes MR. Practical methods for incorporating summary time-toevent data into meta-analysis. Trials. 2007; 8:16.

52. Mihaly Z, Kormos M, Lanczky A, Dank M, Budczies J, Szasz MA and Gyorffy B. A meta-analysis of gene expression-based biomarkers predicting outcome after tamoxifen treatment in breast cancer. Breast cancer research and treatment. 2013; 140(2):219-232.

53. Gyorffy B, Lanczky A and Szallasi Z. Implementing an online tool for genome-wide validation of survivalassociated biomarkers in ovarian-cancer using microarray data from 1287 patients. Endocrine-related cancer. 2012; 19(2):197-208.

54. Li Q, Birkbak NJ, Gyorffy B, Szallasi Z and Eklund AC. Jetset: selecting the optimal microarray probe set to represent a gene. BMC bioinformatics. 2011; 12:474.

55. Mantel $\mathrm{N}$ and Haenszel W. Statistical aspects of the analysis of data from retrospective studies of disease. Journal of the National Cancer Institute. 1959; 22(4):719-748.

56. Higgins JP and Thompson SG. Quantifying heterogeneity in a meta-analysis. Statistics in medicine. 2002; 21(11):15391558.

57. Egger M, Davey Smith G, Schneider M and Minder C. Bias in meta-analysis detected by a simple, graphical test. Bmj. 1997; 315(7109):629-634. 